\title{
Influenza T-cell Epitope-Loaded Virosomes Adjuvanted with CpG as a Potential Influenza Vaccine
}

\author{
Peter C. Soema • Sietske K. Rosendahl Huber • Geert-Jan Willems • Wim Jiskoot • Gideon F. A. Kersten • Jean-Pierre Amorij
}

Received: 18 July 2014 / Accepted: 20 October 2014 / Published online: 25 October 2014

(C) The Author(s) 2014. This article is published with open access at Springerlink.com

\begin{abstract}
Purpose Influenza $\mathrm{CD} 8{ }^{+}$T-cell epitopes are conserved amongst influenza strains and can be recognized by influenza-specific cytotoxic T-cells (CTLs), which can rapidly clear infected cells. An influenza peptide vaccine that elicits these CTLs would therefore be an alternative to current influenza vaccines, which are not cross-reactive. However, peptide antigens are poorly immunogenic due to lack of delivery to antigen presenting cells, and therefore need additional formulation with a suitable delivery system. In this study, the potential of virosomes as a delivery system for an influenza T-cell peptide was investigated.

Methods The conserved human HLA-A2.I influenza T-cell epitope $\mathrm{MI}_{\text {58-66 }}$ was formulated with virosomes. The immunogenicity and protective effect of the peptide-loaded virosomes was assessed in HLA-A2 transgenic mice. Delivery properties of the virosomes were studied in mice and in in vitro dendritic cell cultures. Results Immunization of HLA-A2.I transgenic C57BL/6 mice with peptide-loaded virosomes in the presence of the adjuvant CpG-ODN 1826 increased the number of peptide-specific CTLs. Vaccination with adjuvanted peptide-loaded virosomes reduced weight loss in mice after heterologous influenza infection. Association with fusion-active virosomes was found to be crucial for antigen uptake by dendritic cells, and subsequent induction of CTLs in mice.
\end{abstract}

Electronic supplementary material The online version of this article (doi: | 0. I 007/s I | 095-0 | 4- | 556-3) contains supplementary material, which is available to authorized users.

P. C. Soema $(\bowtie) \cdot$ G.-J. Willems · G. F. A. Kersten • I.-P. Amorij

Intravacc (Institute for Translational Vaccinology)

Antonie van Leeuwenhoeklaan 9, 372 I MA Bilthoven, The Netherlands

e-mail: peter.soema@intravacc.nl

P. C. Soema

e-mail: petersoema@gmail.com

P. C. Soema · W. Jiskoot • G. F. A. Kersten

Division of Drug Delivery Technology, Leiden Academic Centre for

Drug Research (LACDR), Leiden University, Einsteinweg 55

2333 CC Leiden, The Netherlands
Conclusions These results show that influenza virosomes loaded with conserved influenza epitopes could be the basis of a novel cross-protective influenza vaccine.

KEY WORDS cross-protective · cytotoxic T-cells · influenza vaccine $\cdot$ peptide vaccine $\cdot$ virosomes

\section{ABBREVIATIONS}

CpG CpG-ODN 1826

IFA Incomplete Freund's adjuvant

P-V Peptide-loaded virosomes

\section{INTRODUCTION}

The need for cross-protective influenza A vaccines has increased in recent years after several global outbreaks of highly pathogenic influenza A strains, such as avian $\mathrm{H} 5 \mathrm{Nl}$ (1), swine H1N1 and avian H7N9 $(2,3)$. The current, mainly antibodyinducing influenza $\mathrm{A}$ vaccines are generally ineffective against influenza A strains which underwent antigenic shifts and drifts, which leads to vaccine mismatch. Influenza-specific antibodies induced by mismatched vaccines fail to recognize
S. K. Rosendahl Huber

Centre for Infectious Disease Control Netherlands

National Institute for Public Health and the Environment (RIVM)

Antonie van Leeuwenhoeklaan 9, 372 I MA Bilthoven, The Netherlands 
the surface proteins hemagglutinin (HA) and neuraminidase (NA). As a result, the composition of the current influenza vaccines has to be adjusted frequently to cope with these antigenic changes. $\mathrm{CD}^{+}$cytotoxic T-cells (CTLs) that are specific for conserved epitopes of internal influenza A proteins, such as matrix protein and nucleoprotein, may provide cross-protection and are thus unaffected by antigenic changes (4). Influenza-specific CTLs can efficiently clear virus-infected cells, thereby stopping viral replication and spread. Influenzaspecific $\mathrm{CD}^{+}$T-cells induced by influenza infection were recently correlated with less severe illness in adults infected with pandemic $\mathrm{H} 1 \mathrm{~N} 1$ virus (5). Inducing influenza-specific CTLs by vaccination could therefore be a promising approach to achieve cross-protection against heterologous influenza A strains (6).

The influenza $\mathrm{Ml}_{58-66}$ peptide is a highly conserved human major histocompatibility complex (MHC) class I restricted epitope $(7,8)$, which can induce influenza-specific CTLs. However, before $\mathrm{CD}^{+}{ }^{+} \mathrm{T}$-cells can be induced, several critical processes have to take place (9). Delivery of the peptide antigens to antigen-presenting cells (APGs), in particular dendritic cells (DCs), is crucial for antigen presentation on $\mathrm{MHC}$ class I molecules. Therefore, formulation of the peptide antigens with a suitable delivery system, such as virosomes, is required. Influenza virosomes were previously shown to be capable of efficient delivery of peptide antigens and subsequent CTL induction (10). However, virosomal formulations can only deliver low doses of peptide antigens and lack pathogen-associated molecular patterns (PAMPs). The inclusion of immunopotentiators such as Toll-like receptor (TLR) agonists could improve the immunogenicity of the vaccine formulation, without the need to increase the peptide loading efficiency in virosomes, which requires complicated methods (11). While adjuvants have been used to increase neutralizing antibody levels induced by virosomal vaccines (12), their effect on influenza peptidespecific $\mathrm{CD}^{+} \mathrm{T}$-cell responses in combination with virosomes has yet to be determined.

In this study, we investigated the immunogenicity of virosomes loaded with the human HLA-A2.1-restricted peptide $\mathrm{Ml}_{58-66}$, derived from influenza matrix protein 1 . Influenza-specific $\mathrm{CD}^{+}{ }^{+} \mathrm{T}$-cell responses and supporting antibody titers were assessed in HLA-A2.1 transgenic mice after immunization. The addition of TLR 9 agonist CpG was found to be an effective adjuvant for MHC class I restricted peptides in conjunction with virosomes. Furthermore, immunization with peptide-loaded virosomes adjuvanted with $\mathrm{CpG}$ increased the rate of recovery in mice infected with a heterologous influenza strain. Finally, delivery properties of virosomes were extensively characterized in in vitro human DC models and in vivo in transgenic mice. Both peptide association with the virosomes and virosomal cell binding and membrane fusion capabilities were found to be crucial for peptide uptake by DCs and induction of peptide-specific CTLs in mice.

\section{MATERIALS AND METHODS}

\section{Preparation of Virosomes}

Virosomes were prepared from $\beta$-propiolactone inactivated egg-derived influenza A/PR8/34 H1N1 virus as described previously (13). In brief, whole inactivated influenza virus (WIV) was disrupted by the addition of $100 \mathrm{mM} \mathrm{1,2-}$ dihexanoyl-sn-phosphatidylcholine (DHPC, Avanti Polar Lipids) in HNE (5 mM HEPES, $150 \mathrm{mM} \mathrm{NaCl,} 1 \mathrm{mM}$ EDTA, pH 7.3) buffer. Nucleocapsid was removed from the membrane lipids and surface proteins by ultracentrifugation. Virosomes were reconstituted by removal of the detergent by dialysis against HNE buffer. Subsequently, virosomes were purified by centrifugation on a discontinuous sucrose gradient $(10 \% / 60 \%$ \% $/ v$ in $\mathrm{HNE})$, and sucrose was removed by dialysis against HNE buffer. Peptide-loaded virosomes were obtained by adding $125 \mu \mathrm{g} / \mathrm{mL} \mathrm{M1}_{58-66}$ peptide (GILGFVFTL, kindly provided by the Netherlands Cancer Institute) to the virosomes (1:36 peptide:protein $w / w$ ratio) prior to detergent removal to enable peptide encapsulation. As negative control, fusion-inactivated virosomes were prepared. Virosomes were fusion-inactivated after peptide-loading by lowering the buffer $\mathrm{pH}$ to 4.5 with a pretitrated volume of $1 \mathrm{M} \mathrm{HCl}$, and subsequently incubated at $37^{\circ} \mathrm{C}$ for $15 \mathrm{~min}$. Afterwards, $\mathrm{pH}$ was restored to $\mathrm{pH} 7.3$ with a pretitrated volume of $1 \mathrm{M} \mathrm{NaOH}$.

\section{Characterization of Virosome Formulations}

Protein composition of the peptide-loaded virosomes was determined by SDS-PAGE, by using a $12 \%$ precast polyacrylamide gel (Thermo Scientific) and Coomassie Brilliant Blue (Thermo Scientific) staining.

Mean particle size distribution and zeta potential were determined by dynamic light scattering (DLS) with a Malvern Nano ZS (Malvern Instruments). Samples were diluted six fold in MilliQ for zeta potential analysis.

\section{Hemolysis Assay}

Virosome fusion activity was determined by using a hemolysis assay as described previously (14). Vaccine preparations were mixed with human blood erythrocytes and $0.1 \mathrm{M} 2-(\mathrm{N}-$ morpholino)ethanesulfonic acid (MES) buffer with different $\mathrm{pH}$ ranging from 4.5 to 5.5 , and incubated at $37^{\circ} \mathrm{C}$. After allowing fusion for $30 \mathrm{~min}$, the released hemoglobin was quantified in the supernatant after centrifugation by reading absorbance at $540 \mathrm{~nm}$ using a Synergy Mx reader (Biotek). 
Hemoglobin release of erythrocytes mixed with water was set as maximal hemolysis $(100 \%)$.

\section{Association of Peptide to Virosomes}

The interaction between peptides and virosomes was studied using size-exclusion chromatography ( $\mathrm{SEG}$ ). $\mathrm{M1}_{58-66}$ peptide was labeled with fluorescein for detection purposes. Peptideloaded virosomes or peptide mixed with empty virosomes were applied on a pre-washed PD-10 column (GE Healthcare). Samples were eluted with HNE buffer, and fractions of $1 \mathrm{~mL}$ were collected in tubes and subsequently analyzed for peptide and protein content by using fluorescence (exCitation at $480 \mathrm{~nm}$ and emission at $530 \mathrm{~nm}$ ) and Lowry protein assay respectively.

\section{Immunizations}

Animal experiments were performed according to the guidelines provided by the Dutch Animal Protection Act, and were approved by the Committee of Animal Experimentation (DEG) of the National Institute of Public Health and the Environment (RIVM) under registration numbers PO201200156 and PO201300046. 8-12 week old female HLA-A2.1 transgenic C56BL/6 mice (bred and maintained at Intravacc) were used in all studies. Mice received subcutaneous injections $(100 \mu \mathrm{l} /$ dose $)$ in the left hind flank at day 0 and 21 under isoflurane anesthesia. Immunizations consisted of either PBS, peptide mixed with CpG, peptide in 50\% Incomplete Freund's Adjuvant (IFA, Sigma-Aldrich) with CpG, peptide-loaded virosomes (mixed with and without $\mathrm{CpG}$ ), inactivated peptide-loaded virosomes mixed with $\mathrm{CpG}$, and free peptide mixed with empty virosomes and CpG. Mice received $1 \mu \mathrm{g}$ of $\mathrm{M}_{58-66}$ peptide per dose and $180 \mu \mathrm{g}$ of virosomal protein. When mentioned, $50 \mu \mathrm{g}$ of $\mathrm{CpG}-$ ODN 1826 (Invivogen) per dose was used as an adjuvant. As a positive control, one group of mice was infected with $1 \times 10^{3}$ PFU of influenza A/HKx31 H3N2 virus in $50 \mu \mathrm{l}$ PBS by intranasal administration under isoflurane anesthesia. On day 35 animals were sacrificed by bleeding and cervical dislocation under isoflurane anesthesia.

\section{Challenge Study}

For the challenge study, six mice were immunized as described previously. Additionally, one group of mice was immunized twice with $180 \mu \mathrm{g}$ (total protein) of influenza A/PR8 WIV vaccine on the same immunization schedule as previous groups. On day 35, mice were infected with a sublethal dose of $1 \times 10^{5} \mathrm{PFU}$ of influenza $\mathrm{A} / \mathrm{HK} \times 31$ virus in $50 \mu \mathrm{l}$ PBS by intranasal administration. Subsequently, mice were weighed daily until day 14 after challenge, after which the mice were sacrificed.

\section{Intracellular Staining}

Peptide-specific cytotoxic T-cells were quantified by flow cytometry analysis. Single cell suspensions of excised spleens were obtained using $70 \mu \mathrm{m}$ cell strainers (BD Falcon). Subsequently, $2 * 10^{6}$ splenocytes were plated per well in 48 wells culture plates (Greiner) and restimulated with $50 \mathrm{ng} \mathrm{M1} \mathrm{M}_{58-66}$ peptide for $18 \mathrm{~h}$ at $37^{\circ} \mathrm{C} 5 \% \mathrm{CO}_{2}$. Next, Golgi-plug (1:1000, BD) was added to the cells to inhibit cellular protein and cytokine transport, and cells were incubated for another $4 \mathrm{~h}$. Subsequently, cells were transferred to a 96-wells plate, washed twice with FACS buffer (PBS, 0.5\% BSA), and stained with anti-mouse CD4-PE (BD Biosciences), anti-mouse CD8-FITC (BD Biosciences) and Live/deadAqua (Invitrogen). Next, cells were washed twice with FACS buffer, and fixed with fixation-permeabilization buffer (BD Biosciences). Subsequently cells were washed with permeabilization wash buffer (BD Biosciences), and intracellular staining was performed with IFN- $\gamma$-APG (Biolegend). Finally, cells were washed with FACS buffer and 1.5 to 2 million cells were measured on a FACS Canto II flow cytometer (BD Biosciences). Data was analyzed using FlowJo software version 9 (Tree Star) for Mac OSX.

\section{Enzyme Linked Immunosorbent Spot Assay (ELISpot)}

Cytokines produced by spleen cells were determined by ELISpot. 96-wells Multiscreen PVDF filter plates (Millipore) were activated by adding $25 \mu \mathrm{L} 70 \%$ ethanol for $2 \mathrm{~min}$, and subsequently washed three times with PBS. Plates were coated overnight with anti-mouse IFN- $\gamma$ antibodies (U-Cytech) at $4^{\circ} \mathrm{C}$. Next, filter plates were washed three times and blocked with $5 \%$ Hyclone fetal calf serum (FCS, Thermo Scientific) for $1 \mathrm{~h}$ at $37^{\circ} \mathrm{C}$. Subsequently, $4^{*} 10^{5}$ isolated spleen cells in IMDM medium, $5 \%$ FCS were added to each well with or without $50 \mathrm{ng} \mathrm{M1} 1_{58-66}$ peptide, and incubated overnight at $37^{\circ} \mathrm{C}$. After overnight stimulation, filter plates were washed five times and IFN- $\gamma$ was detected using biotinylated antimouse antibodies (U-Cytech) and $100 \mu \mathrm{L}$ BCIP/NBT reagent (Thermo Scientific) per well. Spots were allowed to develop for $15 \mathrm{~min}$ after which the plates were thoroughly washed with tap water. Spots were counted using an A.EL.VIS ELISpot reader (Aelvis). The number of IFN- $\gamma$ producing cells in antigen stimulated spleen cells was obtained after background correction (subtracting number of spots produced by splenocytes incubated with medium).

\section{Hemagglutination Inhibition Assay}

Hemagglutination-inhibition (HI) titers in mouse sera were determined by a HI assay. Sera were treated overnight with diluted receptor-destroying enzyme from Vibrio cholerae (1:5, Sigma-Aldrich) at $37^{\circ} \mathrm{C}$ to remove non-specific inhibitors, and 
were subsequently inactivated at $56^{\circ} \mathrm{C}$ for $30 \mathrm{~min}$. Finally, PBS was added to the sera to obtain a 1:10 dilution. Diluted sera were serially diluted two-fold with PBS. Four hemagglutinating units of inactivated influenza A/PR/8/34 or influenza $\mathrm{A} / \mathrm{HK} 31$ were subsequently added to each well and incubated for $20 \mathrm{~min}$ at room temperature after mixing. Next, an equal amount of a $0.5 \%(v / v)$ turkey erythrocyte suspension was added to the wells and incubated for $45 \mathrm{~min}$ at room temperature. HI titers are reported as the reciprocal of the highest serum dilution capable to completely prevent hemagglutination.

\section{Enzyme Linked Immunosorbent Assay (ELISA)}

Influenza A/PR8 and A/HKx31-specific antibody titers were determined by ELISA as described previously (15). In short, Microlon 96-wells flatbottom plates (Greiner) were coated overnight with either $600 \mathrm{ng}$ (HA) of A/PR8/34 subunit or A/HKx31 virus per well at $4^{\circ} \mathrm{C}$. Serial two-fold dilutions of individual mouse sera in PBS, 0.5\% BSA, 0.1\% Tween80 were applied on the plate and incubated for $1 \mathrm{~h}$ at $37^{\circ} \mathrm{C}$. Subsequently, plates were incubated for $1 \mathrm{~h}$ at $37^{\circ} \mathrm{C}$ with horseradish peroxidase-conjugated goat antibodies against mouse IgG, IgG1 or IgG2c (1:5000, Southern Biotech). Detection of antibodies was performed with TMB substrate buffer $(0.4 \mathrm{mM}$ TMB in $0.11 \mathrm{M}$ sodium acetate, $0.006 \%$ $\mathrm{H}_{2} \mathrm{O}_{2}, \mathrm{pH}$ 5.5). The reaction was stopped by adding $2 \mathrm{M}$ sulfuric acid, after which the optical density (OD) was measured at a wavelength of $450 \mathrm{~nm}$ by using a Synergy Mx platereader (BioTek). Titers are reported as the reciprocal of the serum dilution corresponding to $\mathrm{OD}_{450}=0.2$ after background correction.

\section{Dendritic Cell Uptake Studies}

Peptide antigen uptake by DCs was determined as follows. Fresh blood was collected from healthy donors and collected in heparin-coated tubes. Peripheral blood mononuclear cells (PBMCs) were isolated from whole blood using a Lymphoprep (Axis-Shield) gradient. Subsequently, CD14 ${ }^{+}$ monocytes were isolated from the PBMC fraction by labeling with human CD14 MicroBeads (Miltenyi Biotec) and subsequent separation with a magnetic LS MACS column (Miltenyi Biotec). Finally, monocytes were plated at a concentration of $0.4 * 10^{6}$ cells $/ \mathrm{mL}$ in 48 -wells plates in IMDM medium (Invitrogen) containing 1\% FCS, 500 U/mL GM-CSF (Peprotech) and $800 \mathrm{U} / \mathrm{mL}$ IL-4 (Sanquin). After 6 days, vaccine formulations were incubated for 4, 24 and $48 \mathrm{~h}$ with the immature DCs at a concentration of $50 \mathrm{ng} / \mathrm{mL} \mathrm{M1}_{58-66^{-}}$ FITC peptide in IMDM with $500 \mathrm{U} / \mathrm{mL}$ GM-CSF. Subsequently, cells were transferred to a 96-wells plate, washed twice with FACS buffer, and stained with Live/dead-Aqua. Cells were washed twice with FACS buffer and analyzed on a FACS Canto II flow cytometer. Data was analyzed by using FlowJo 9 software for Mac OSX. Peptide uptake is reported as mean fluorescent intensity (MFI) of the FITC signal.

\section{Statistics}

Data were analyzed by using one-way ANOVA with TukeyKramer's method for multiple comparisons. Probability (p) values of $p \leq 0.05$ were considered statistically significant. Statistics were performed by using Graphpad Prism Software version 6.03 for Windows.

\section{RESULTS}

\section{Characteristics of Peptide-Loaded Virosomes}

To confirm that virosome production was successful, the protein composition of peptide-loaded virosomes was analyzed by SDS-PAGE. We observed that peptide-loaded virosomes $(\mathrm{P}-\mathrm{V})$ retained $\mathrm{HA}$ and NA proteins, whereas internal proteins, such as matrix protein 1 (M1), were removed from the virosome particles (Fig. 1). Additionally, SDS-PAGE analysis of $\mathrm{P}-\mathrm{V}$ under reducing conditions revealed that subunits $\mathrm{HA}_{1}$ and $\mathrm{HA}_{2}$ from $\mathrm{HA}$ mono- and dimers were formed, similar to WIV. Dynamic light scattering showed a particle size of $140 \pm 2 \mathrm{~nm}$ (mean $\pm \mathrm{SD}, n=3$ ) for $\mathrm{P}-\mathrm{V}$, which was

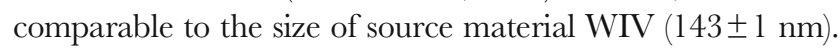
The polydispersity index (PDI) of P-V was 0.121 , indicating that the particle distribution was very homogeneous and comparable to the PDI of WIV (0.036). The zeta potential of virosomes was $-21.2 \pm 1.7 \mathrm{mV}$ (mean $\pm \mathrm{SD}, n=3$ ), which

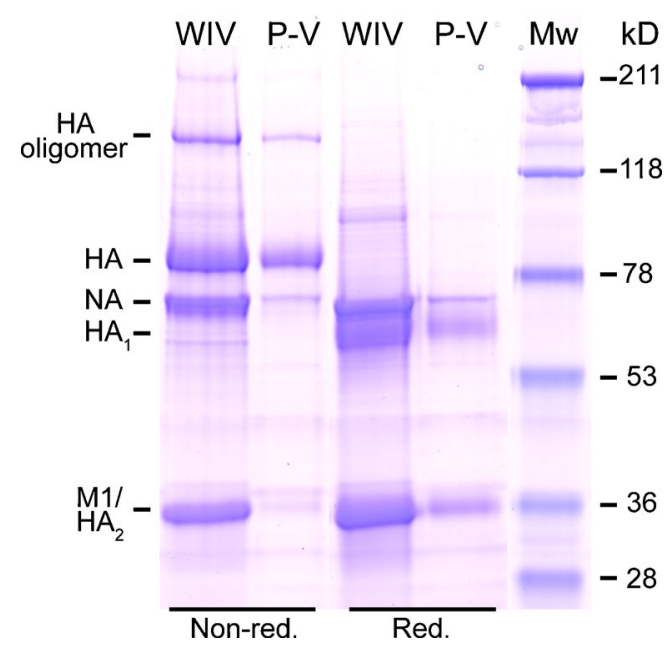

Fig. I Protein profile of peptide-loaded virosomes. SDS-PAGE analysis of peptide-loaded virosomes (P-V) and source material whole inactivated influenza virus (WIV) under non-reduced and reduced conditions on a 12\% precast gel stained with Coomassie Brilliant Blue. Protein identities were confirmed by mass spectrometry. 


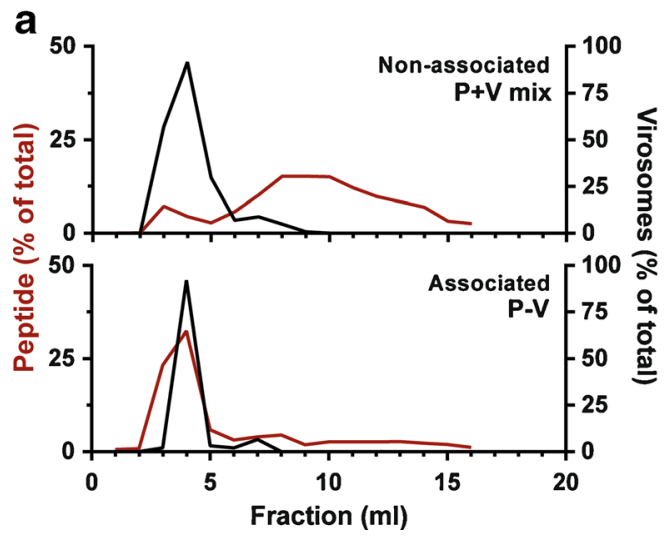

b

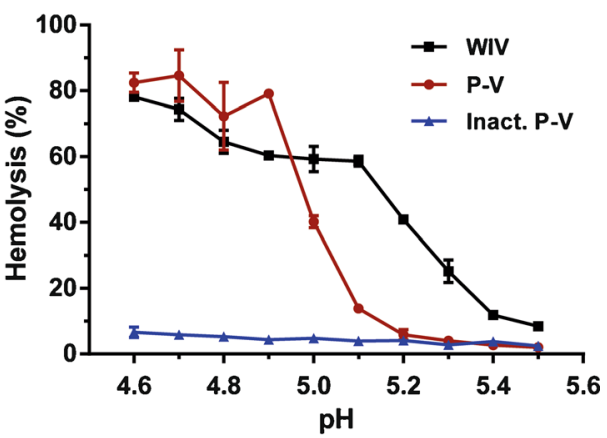

Fig. 2 Characteristics of peptide-loaded virosomes. Peptide association of peptide mixed with virosomes (P+V mix) and peptide-loaded virosomes (P-V) analyzed by size exclusion chromatography (a). Black lines show the virosome elution pattern (based on protein determination), whereas the red line shows the elution of peptide (based on fluorescence of $\mathrm{MI}_{58-66}$-FITC). The fusogenic activity of WIV (black), P-V (red) and fusion-inactivated P-V (blue) was determined between $\mathrm{pH} 4.6$ and 5.5 by a hemolysis assay $(\mathbf{b})$. Data represents mean $\pm \mathrm{SD}(n=3)$.

was similar to that of WIV $(-21.5 \pm 0.3 \mathrm{mV})$. Therefore, the peptide-loaded virosomes closely resembled WIV particles in terms of particle size and surface charge, but were enriched in $\mathrm{HA}$ and NA.

Size-exclusion chromatography confirmed that simple mixing of peptide and virosomes did not result in substantial association between the two components (Fig. 2a). As expected, both peptide and virosomes co-eluted when peptideloaded virosomes were applied onto the SEC column, indicating association. The association efficiency of the peptide with the virosomes was typically $4-6 \%$ of the total amount of added peptide.

In order to confirm whether P-V still possessed fusion activity, a hemolysis assay was performed. Both peptideloaded virosomes and WIV showed low-pH induced fusion activity in the $\mathrm{pH}$ range that is representative for the endosome (Fig. 2b). Additionally, P-V were shown to be successfully fusion-inactivated by short (15 min) exposure to $\mathrm{pH} 4.5$. P-V fused at slightly lower $\mathrm{pH}$ compared to WIV, which might be caused by small differences in HA confirmation and stability in peptideloaded virosomes.

\section{Immunogenicity of Peptide-Loaded Virosomes in HLA-A2 Transgenic Mice}

To assess whether the produced peptide-loaded virosomes were able to induce peptide-specific T-cell responses, HLAA2.1 transgenic C57BL/6 mice were primed and boosted three weeks after priming with either peptide-loaded virosomes or P-V adjuvanted with $\mathrm{CpG}$. PBS and peptide mixed with IFA and $\mathrm{CpG}$ were administered as negative and positive control, respectively. Peptide-specific T-cell responses in restimulated splenocytes were determined 2 weeks after booster vaccination. Flow cytometry analysis revealed that splenocytes from mice immunized with peptide-loaded virosomes contained peptide-specific IFN- $\gamma^{+} \mathrm{CD}^{+}{ }^{+} \mathrm{T}$-cells after $e x$ vivo stimulation (Fig. 3a), as opposed to PBS injected mice. P-V were able to induce peptide-specific IFN- $\gamma^{+} \mathrm{CD}^{+}$ T-cell levels comparable to the levels induced by $\mathrm{P}$ mixed with IFA and CpG. The addition of CpG to P-V significantly $(p<0.001)$ increased the number of peptide-specific $\mathrm{CD}^{+}$ T-cells, which confirmed the immunopotentiating effect of CpG. The increase of $\mathrm{IFN}^{-}{ }^{+} \mathrm{CD} 8^{+}$T-cells after adding $\mathrm{CpG}$ to the P-V formulation was in line with the increased frequency of IFN- $\gamma$ producing cells observed utilizing an ELISPOT assay (Fig. 3b). In order to investigate the contribution of viral material to the T-cell response, mice were immunized with WIV mixed with peptide and CpG. Flow cytometry (Supplementary Figure S1A) and ELISPOT (Supplementary Figure S1B) assays confirmed that WIV induced minimal peptide-specific T-cells.

While virosomes mainly act as an efficient vehicle to deliver the peptide antigen to the APCs, the presence of $\mathrm{CD}^{+}{ }^{+} \mathrm{T}$-cell epitopes in the sequence of virosomal surface proteins enable virosomes to provide helper $\mathrm{T}$-cell $\left(\mathrm{T}_{\mathrm{H}}\right)$ responses. $\mathrm{T}_{\mathrm{H}}$ responses are able to support the induction of $\mathrm{CD}^{+} \mathrm{T}$-cells (16), and $\mathrm{CD} 4^{+} \mathrm{T}$-cell epitopes are thus an important part of the vaccine formulation. To gain further insight into the possible T-cell help that virosomes and the adjuvant provide, the $\mathrm{T}_{\mathrm{H} 1} / \mathrm{T}_{\mathrm{H} 2}$ balance was assessed by determination of $\operatorname{IgG} 1$ and IgG2c isotype titers induced by P-V with or without $\mathrm{CpG}$. The results show that IgG1 titers remained unchanged (Fig. 3c) after addition of $\mathrm{CpG}$, but IgG2c titers were significantly $(p<0.0001)$ increased after vaccination with $\mathrm{CpG}$ adjuvanted P-V when compared to P-V alone (Fig. 3d). This indicates that addition of $\mathrm{CpG}$ to $\mathrm{P}-\mathrm{V}$ skewed influenza specific responses towards a $\mathrm{T}_{\mathrm{H} 1}$ response, which supports the $\mathrm{CD}^{+}{ }^{+} \mathrm{T}$-cell response against the influenza peptide.

In addition to the T-cell epitopes, the P-V formulations contain virosomal B-cell epitopes (mainly on surface antigens HA and NA) that induce influenza-specific antibodies. While 

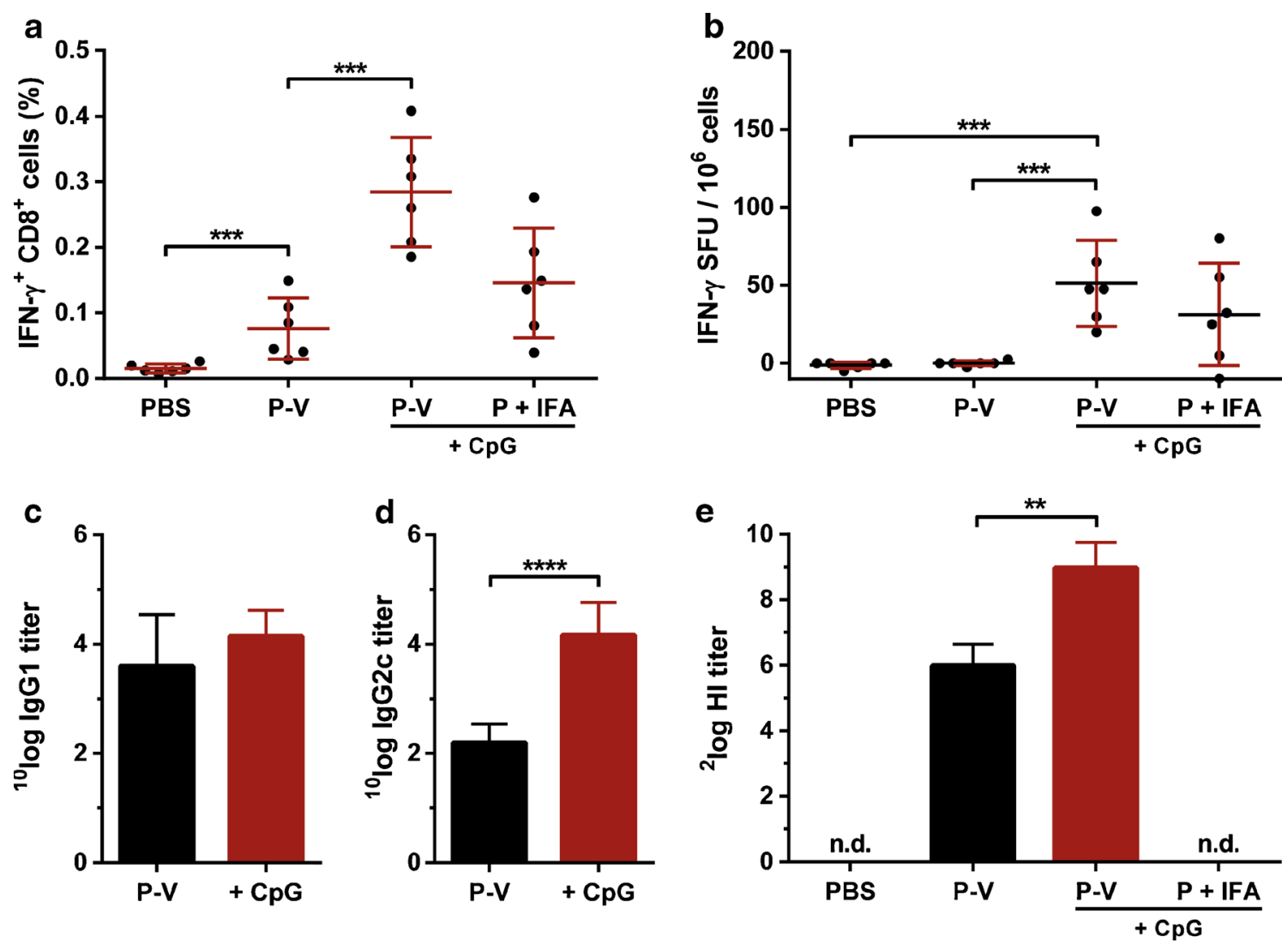

Fig. 3 Immunogenicity of (non-)adjuvanted peptide-loaded virosomes. HLA-A2.I transgenic C57BL/6 mice were immunized twice with I $\mu \mathrm{g}$ of $\mathrm{MI}{ }_{58-66}$ peptide formulated in either virosomes (P-V), virosomes adjuvanted with CpG (P-V + CpG) or Incomplete Freund's Adjuvant (IFA) with CpG (P + IFA + CpG). Mice were immunized with PBS as negative control. Two weeks after the final vaccination, peptide-specific CD8+ T-cell responses in ex vivo stimulated splenocytes were determined using flow cytometry $(\mathbf{a})$ and ELISPOT (b). Antibody isotypes $\operatorname{lgG}$ I (c) and IgG2c (d) titers were determined in mice sera. HI titers

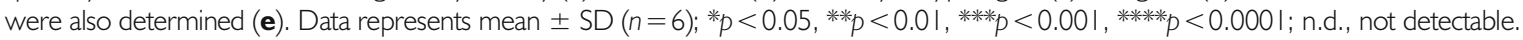

these antibodies are usually not cross-reactive, they do provide protection against homologous influenza strains. Thus, in case the circulating virus matches the source influenza strain of the virosomes, additional humoral responses can aid in protection. CpG adjuvanted P-V induced significantly $(p<0.01)$ higher HI titers compared to non-adjuvanted P-V (Fig. 3e). Total IgG titers showed a similar significant $(p<0.05)$ increase after addition of $\mathrm{CpG}$ to the P-V (Supplementary Figure $\mathrm{S} 1 \mathrm{C}$ ). This underlines that $\mathrm{CpG}$ is not only an effective adjuvant for $\mathrm{T}$-cell induction, but also improves B-cell responses, as observed previously (12). As expected, there were no detectable $\mathrm{HI}$ and total IgG titers in sera of control mice receiving PBS or peptide mixed with IFA and CpG, due to the lack of influenza surface antigens in these formulations.

\section{Efficacy of Peptide-Loaded Virosomes Against Heterologous Influenza Infection in Mice}

In addition to the assessment of immunological responses, the efficacy of the $\mathrm{P}-\mathrm{V}$ vaccine against heterologous influenza infection was examined. HLA-A2.1 transgenic C57BL/6 mice were immunized with the vaccine and subsequently infected with a sublethal dose of influenza HKx31 (H3N2) virus. The weight of the infected animals was monitored for 14 days (Fig. 4). Mice that were previously infected with a small dose of live influenza HKx31 did not show any weight loss after challenge. Mice that received either PBS, WIV or peptide mixed with IFA and CpG showed a decline in weight until day 7 , after which the animals recovered slowly. In contrast, mice immunized with $\mathrm{CpG}$ adjuvanted P-V started to recover already after day 6 , and their total weight loss was less severe than that of mice immunized with PBS or peptide mixed with IFA and CpG. Moreover, at day 7 and 8, the weight of mice immunized with $\mathrm{P}-\mathrm{V}$ adjuvanted with $\mathrm{CpG}$ was not statistically different than the weight of protected mice pre-exposed to $\mathrm{HKx} 31$, whereas mice immunized with PBS, WIV or peptide with IFA and CpG did show a significant $(p<0.0001)$ difference with protected mice. HKx31-specific $\mathrm{IgG}$ and $\mathrm{HI}$ determination showed that $\mathrm{P}-\mathrm{V}$ induced minimal amounts of HKx31-specific antibodies, which did not possess any neutralizing capacity (Supplementary Figure S2). Thus, this indicates that the increased recovery was not mediated by cross-reactive neutralizing antibodies, but rather by the 


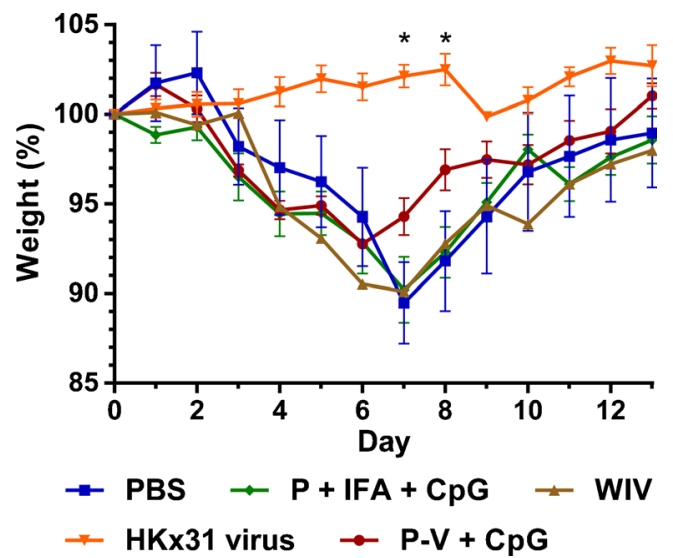

Fig. 4 Efficacy of vaccine after sublethal heterologous influenza infection. HLA-A2.I transgenic C57BL/6 mice were immunized twice with either peptide-loaded virosomes with CpG (red), peptide mixed with incomplete Freund's adjuvant and CpG (green), WIV (brown) or PBS (blue). As a positive control, mice were challenged with a sublethal dose of $H K \times 3 I$ virus. Subsequently, mice were infected with heterologous HKx3 I (H3N2) influenza virus and their weight was monitored for 14 days. Data represents mean \pm SEM $(n=6) ;{ }^{*} p<0.000$ I for PBS and P + IFA $+C p G$ groups versus HK×3 I.

increase of $\mathrm{CD}^{+}{ }^{+} \mathrm{T}$-cells (and/or $\mathrm{CD} 4^{+} \mathrm{T}$-cells). Vaccination with $\mathrm{P}-\mathrm{V}$ mixed with $\mathrm{CpG}$ may therefore contribute to the recovery from influenza after heterologous influenza infection.

\section{Influence of Association Between Peptide and Virosomes on Peptide Association with Dendritic Cells and $\mathrm{CDB}^{+} \mathrm{T}$-cell Responses}

In order to gain some mechanistic insight into the mode of action of our P-V vaccine, we investigated the importance of peptide association with the virosomes for the induction of peptide-specific CTLs. The delivery concept of virosomes and the importance of peptide association was first assessed in vitro. The uptake of peptide antigens by immature DCs was determined in vitro for $\mathrm{P}-\mathrm{V}$ and free peptide mixed with empty virosomes $(\mathrm{P}+\mathrm{V}$, Fig. 5a). After $4 \mathrm{~h}$ of incubation, $\mathrm{P}-\mathrm{V}$ showed a significantly $(p<0.01)$ higher peptide association with DCs compared to $\mathrm{P}+\mathrm{V}$ or free peptide without any carrier. This trend was also observed after 24 and $48 \mathrm{~h}$ of incubation, resulting in even larger $(p<0.001)$ differences between $\mathrm{P}-\mathrm{V}$ and the other formulations. Incubation of P-V with DCs at a temperature of $4^{\circ} \mathrm{C}$ also showed a significant increase of peptide signal (data not shown), indicating that $\mathrm{P}-\mathrm{V}$ readily associated with the cell membrane of DCs prior to internalization. This indicates that the virosomes attached themselves to the DC surface most probably by sialic acid binding. Thus, association of the peptide with the virosomes positively affects the antigen association with DCs, proving that virosomes act as a delivery system for the peptide antigen.

In addition to the in vitro DG studies, in viwo studies in HLAA2.1 transgenic C57BL/6 mice revealed that association of the peptide with the virosomes is crucial for the induction of
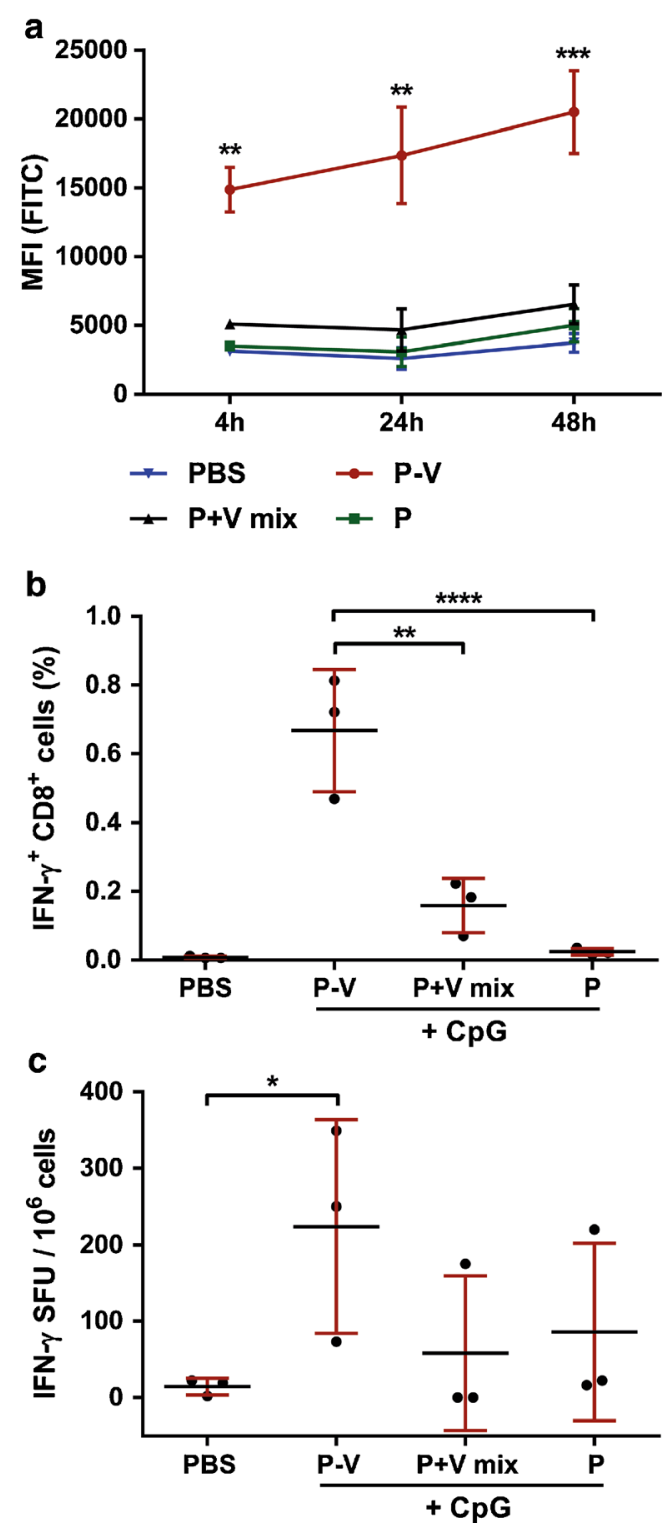

Fig. 5 Influence of peptide-virosome association on in vitro dendritic cell uptake and in vivo T-cell responses. $\mathrm{MI}_{58-66}$ - FITC peptide uptake by human immature DCs was determined by flow cytometry (a). Either PBS, peptideloaded virosomes $(\mathrm{P}-\mathrm{V})$, peptide mixed with empty virosomes $(\mathrm{P}+\mathrm{V}$ mix $)$ or free peptide $(P)$ were incubated at $37^{\circ} \mathrm{C}$ with immature DCs for 4,24 and $48 \mathrm{~h}$. Data represents mean $\pm \mathrm{SD}(n=3)$ performed with DCs obtained from three different donors. HLA-A2.I transgenic C57BL/6 mice were immunized twice with aforementioned formulations adjuvanted with $\mathrm{CpG}$. Peptide-specific CD8 ${ }^{+}$T-cell responses were determined in ex vivo stimulated splenocytes by flow cytometry $(\mathbf{b})$ and ELISPOT (c). Data represents mean \pm $\mathrm{SD}(n=3)$ and is representative of three individual experiments. ${ }^{*} p<0.05$,

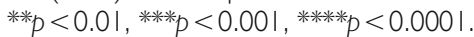

peptide-specific CTLs. CD8 ${ }^{+}$T-cell responses were determined in in vitro stimulated splenocytes two weeks after the boost vaccination (Fig. $5 b)$. P-V + CpG induced significantly $(p<0.0001)$ higher CTL numbers in mice than the peptide + CpG mixture. When CpG adjuvanted peptide was mixed with empty virosomes, the number of peptide-specific CTLs in the spleen was significantly $(p<0.01)$ lower compared to $\mathrm{P}-\mathrm{V}+$ 
CpG. The frequency of IFN- $\gamma$ producing cells showed a similar trend; only P-V + CpG showed increased IFN- $\gamma$ spot-forming units compared to peptide mixed with empty virosomes or free peptide alone (Fig. 5c). This suggests that addition of the adjuvant alone to the $\mathrm{M}_{58-66}$ peptide is not sufficient to induce peptide-specific T-cell responses. Furthermore, association of the peptide with virosome was not critical for the humoral responses or the $\mathrm{T}_{\mathrm{H} 1} / \mathrm{T}_{\mathrm{H} 2}$ balance (Supplemental Figure $\mathrm{S} 3$ ), suggesting that these particular responses are only influenced by the virosome and the presence of an adjuvant.
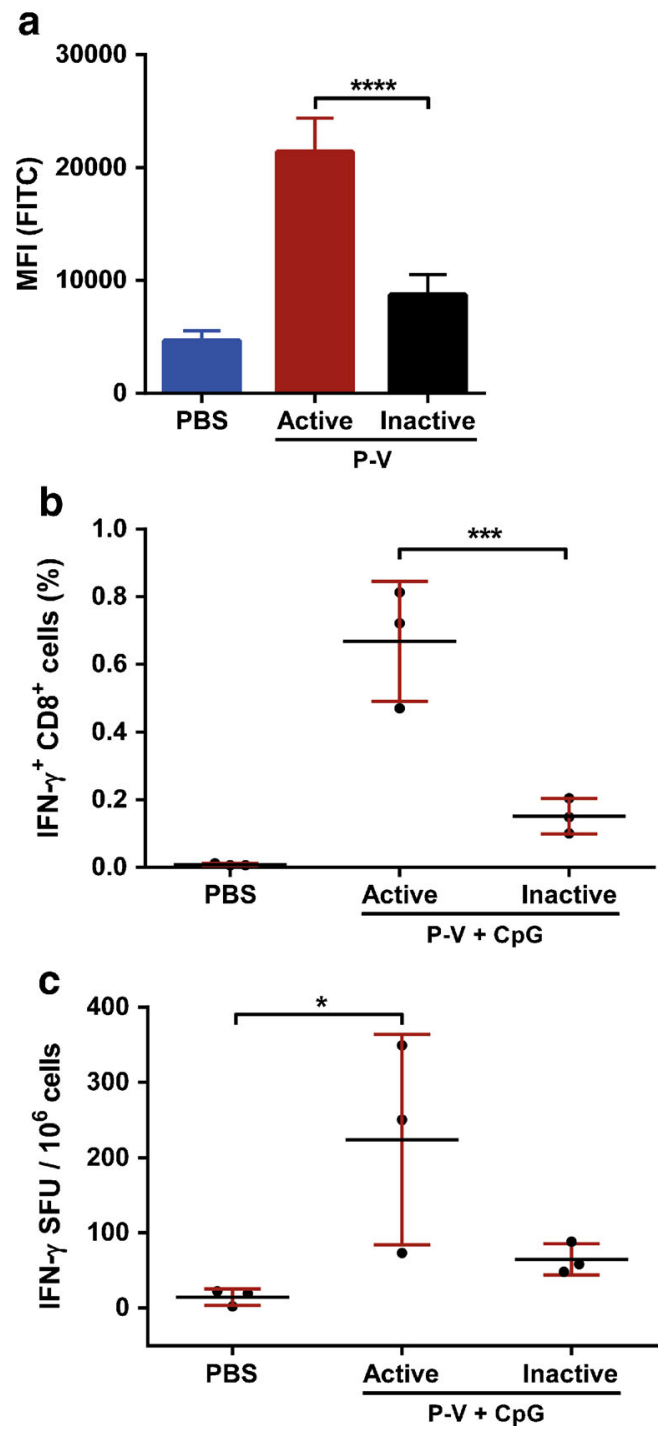

Fig. 6 Impact of loss of fusogenic activity on immunogenicity of peptideloaded virosomes. $\mathrm{MI}_{58-66}$-FITC peptide uptake by human immature DCs was quantified for both fusion-active and inactive P-V after $16 \mathrm{~h}$ of incubation at $37^{\circ} \mathrm{C}(\mathbf{a})$. Data represents mean $\pm \mathrm{SD}(n=3)$ performed with DCs obtained from three different donors. HLA-A2.I transgenic C57BL/6 mice were immunized twice with peptide-loaded virosomes with CpG (Active P-V + CpG) or fusion-inactivated peptide-loaded virosomes with CpG (Inactive P-V $+\mathrm{CpG}$ ). Peptide-specific CD8 ${ }^{+}$T-cells were subsequently quantified in ex vivo stimulated splenocytes by flow cytometry (b) and ELISPOT (c). Data represents mean $\pm \mathrm{SD}(n=3)$ and is representative of three individual experiments. ${ }^{*} p<0.05$, ${ }^{*} * * p<0.001$, $* * * * * 0.0001$

\section{Influence of Virosomal Cell Binding and Membrane Fusion Capabilities on the Immunogenicity of Peptide-Loaded Virosomes}

In addition to the role of peptide association, the role of virosomal cell binding and membrane fusion activity in the immunogenicity of peptide-loaded virosomes was studied. Content of virosomes is released into the cytosol of APCs through $\mathrm{pH}$-mediated fusion with the endosomal membrane. First, peptide association by DCs was quantified by flow cytometry after $16 \mathrm{~h}$ of incubation with (inactivated) vaccine formulations (Fig. 6a). When P-V were fusion-inactivated, the association of peptide decreased significantly $(p<0.0001)$ compared to fusion-active P-V. This indicates that the inherent cell binding and membrane fusion capabilities of virosomes are needed to ensure efficient uptake by APGs.

Next, we investigated whether the limited uptake of peptide by DCs had an impact on T-cell induction after administering inactivated P-V to mice. HLA-A2.1 transgenic C57BL/6 mice immunized with fusion-inactivated $\mathrm{P}-\mathrm{V}$ generated significantly $(p<0.001)$ less peptide-specific $\mathrm{CD}^{+}{ }^{+} \mathrm{T}$-cells than mice immunized with fusogenic P-V (Fig. 6b). The frequency of IFN- $\gamma$ producing cells also showed a decreasing trend after inactivation (Fig. 6c), showing that inactivation of the influenza virosomes has a significant negative impact on the immunogenicity of $\mathrm{Ml}_{58-66}$ peptide associated with the virosome.

To assess the impact of fusion inactivation on the ability of $\mathrm{P}-\mathrm{V}$ to induce humoral responses, influenza-specific antibody titers were determined in serum. The fusion-inactivated formulation induced a significantly $(p<0.0001)$ lower influenzaspecific neutralizing antibody response than the fusion-active P-V (Supplemental Figure S4A). However, the total serum IgG titers were only slightly but significantly $(p<0.01)$ lower for the group receiving fusion-inactivated P-V (Supplemental Figure S4B). This indicates that HA-specific antibodies were unable to inhibit hemagglutination, which could be the effect of reduced antibody avidity or the generation of antibodies recognizing different epitopes. Furthermore, fusioninactivation of P-V did not affect IgG1 titers, but did negatively affect IgG2c titers (Supplemental Figure S3C and S3D), which indicates a shift to a $\mathrm{T}_{\mathrm{H} 2}$ response.

\section{DISCUSSION}

Gurrent research on universal influenza vaccines is directed at targeting conserved parts of the influenza virion. Aside from B-cell epitopes that can induce broadly-protective neutralizing antibodies, a T-cell component is considered to be an important component of future influenza vaccines (17). Influenzaspecific CTLs can increase viral clearance and limit morbidity across multiple influenza strains; moreover, recent studies indicate that cellular responses might be a correlate of 
protection against pandemic influenza strains $(5,18)$. Inducing potent immune responses against influenza-specific T-cell epitopes, however, is challenging due to the nature of the antigen. Subunit (peptide) vaccines generally possess poor immunogenicity due to the lack of any particulate structure or presence of PAMPs. Virosomes have proven to be an efficient delivery system for peptides in previous studies $(10,11,19,20)$, but generally have low peptide association or encapsulation rates. This makes proper dosage of the peptide antigen difficult; if encapsulation rates are low, a disproportionate amount of virosome material is present in the vaccine. Several alternative production methods have been developed that enhanced peptide encapsulation efficiencies. These included chimeric virosomes (11), virosome lyophilization and subsequent reconstitution (19), or covalent attachment of the peptide (20). However, these methods complicate the production process, and might not be suitable for every virosomal peptide formulation. Thus, the addition of an adjuvant to virosomal peptide formulations could be a viable alternative to raise the immunogenicity of the peptide, without increasing peptide and virosome dose or altering the formulation process.

The selected $\mathrm{M1}_{58-66}$ peptide epitope is restricted to the human HLA-A2 serotype. To produce a vaccine that is effective in a global population, several peptide epitopes covering all the HLA serotypes must be included to ensure acceptable coverage. Since few (animal) models currently exist to screen for the various HLA types, we selected a HLA-A2 epitope, which is a common serotype in the Caucasian population and can be tested in HLA-A2.1 transgenic C57BL/6 mice. Thus, in contrast to other preclinical peptide-based vaccine concepts, this concept influenza vaccine could be used directly in humans without changing the peptide antigen.

We demonstrated that the addition of $\mathrm{CpG}$ as an adjuvant significantly increased cellular responses in mice immunized with peptide-loaded virosomes. Additionally, $\mathrm{CpG}$ skewed antibody responses induced by peptide-loaded virosomes towards the IgG2c isotype. The production of IgG2c antibodies is stimulated during $\mathrm{T}_{\mathrm{H} 1}$ type responses (21), which support the induction of $\mathrm{CD}^{+}{ }^{+} \mathrm{T}$-cells (16), which in turn is associated with influenza virus clearance (22). Since our current peptideloaded virosome production process was inefficient, we opted to mix $\mathrm{CpG}$ with our formulation, rather than incorporating it in the virosome, which was previously performed with an avian virosome vaccine (12). Incorporating both $\mathrm{CpG}$ and peptide antigen in a virosome potentially could increase immunogenicity due to the simultaneous signal delivery of both adjuvant and antigen. This would be a logical next step for future research.

The interaction between the peptide antigen and the virosome particles was shown to be an important factor for the overall efficacy of the peptide-loaded virosome vaccine, which confirmed an earlier report (10). Additionally, antigen uptake studies with DGs revealed that association of the antigen with the carrier is important for antigen uptake by APCs. While SEC analysis showed that the peptide was indeed associated with the virosomes, the exact localization of the peptide, e.g. in the aqueous inner compartment or the lipid membrane, remains unknown. The localization of peptide antigens in virosomes could have an impact on presentation on MHC-I molecules by APCs (23), which in turn can affect the quality of the immune response, and is therefore a relevant topic for future studies.

Hemagglutinin conformational integrity and activity, mediating virosomal cell binding and membrane fusion, were shown to be crucial for the induction of $\mathrm{CD}^{+}{ }^{+} \mathrm{T}$-cell responses. In addition to an earlier report which indicated that fusion activity might affect CTL responses induced by $\mathrm{NP}_{147-}$ 155 peptide-loaded virosomes (10), we demonstrated that virosome fusion inactivation had a profound impact on the capacity of virosomes to deliver peptide to DCs, and on the induction of peptide-specific T-cell responses by peptideloaded virosomes. The role of fusion activity is not only important for binding and cell entry of virosomes, but also for CTL induction by WIV vaccines $(24,25)$. Furthermore, fusion-inactivation impaired the induction of influenzaspecific IgG2c antibodies, which could affect helper T-cell responses. In addition, fusion-inactivation impaired the neutralizing ability of influenza-specific antibodies generated after vaccination with peptide-loaded virosomes significantly, while total influenza-specific IgG levels only were reduced slightly.

To assess the efficacy of the vaccine, HLA-A2.1 transgenic C57BL/6 mice were immunized with CpG-adjuvanted P-V and subsequently challenged with a sublethal heterologous HKx31 influenza infection. The mouse weight loss data shown in this study indicate that the elevated numbers of influenzaspecific CTLs after vaccination contributed to the recovery of the mice after heterologous influenza infection, independent of neutralizing antibodies. An increase of circulating $\mathrm{CD}^{+}{ }^{+} \mathrm{T}$ cells might however not be enough to provide complete protection against influenza infections. A boost in $\mathrm{CD}^{+} \mathrm{T}$-cells may help clear the virus and improve the rate of recovery of the mice after infection, but is arguably insufficient to prevent the early onset and spread of infection. Slütter et al. recently showed the importance of local memory $\mathrm{CD}^{+}{ }^{+} \mathrm{T}$-cells in the upper respiratory tract to combat influenza A infections in the early stages (26). This insight implicates that the local induction of respiratory $\mathrm{CD}^{+} \mathrm{T}$-cells could be an important goal for further T-cell based influenza vaccine development. The increased systemic T-cell levels reported in clinical studies might be an indication that elevated local influenza-specific $\mathrm{CD}^{+} \mathrm{T}$ cells in the lungs can provide accelerated recovery and decreased morbidity in influenza-infected patients $(5,27)$.

Aside from $\mathrm{CD}^{+}{ }^{+} \mathrm{T}$-cell responses, it has been established that T-cell help (in the form of $\mathrm{CD}^{+}{ }^{+} \mathrm{T}$-cells) and $\mathrm{B}$-cell responses should not be overlooked (28), and therefore a vaccine concept that utilizes both $\mathrm{T}$-cell and $\mathrm{B}$-cell responses 
should be pursued to obtain a universal influenza vaccine (29). Influenza virosomes could be an excellent candidate platform for a cross-protective influenza vaccine, as it is an effective peptide delivery system and a natural carrier of $\mathrm{CD} 4^{+} \mathrm{T}$-cell and B-cell epitopes.

In conclusion, we demonstrated that peptide-loaded virosomes are able to induce peptide-specific $\mathrm{CD}^{+} \mathrm{T}$-cells. The addition of $\mathrm{CpG}$ as an adjuvant further increased the efficacy of peptide-loaded virosomes. Aside from a greater number of peptide-specific $\mathrm{CD}^{+} \mathrm{T}$-cells, $\mathrm{CpG}$ adjuvanted $\mathrm{P}-\mathrm{V}$ also induced T-cell help and influenza-specific antibodies. Peptide-virosome association and virosome fusion activity are important factors for the effectiveness of P-V. The synergistic effect of virosome particles, fusion activity and $\mathrm{CpG}$ make a potent combination to increase the immunogenicity of peptide antigens. Thus, peptide-loaded virosomes are a promising approach for the development of a cross-protective influenza vaccine.

\section{ACKNOWLEDGMENTS AND DISCLOSURES}

The authors thank Wichard Tilstra, Klaas van Twillert, Dirk Elberts, Nynke Tichelaar and Jolanda Rigters from Intravacc for their assistance with the animal studies. Furthermore, we acknowledge the blood donors for their contribution. This work was supported by the Center for Translational Molecular Medicine grant AMPVACS.

Open Access This article is distributed under the terms of the Creative Commons Attribution License which permits any use, distribution, and reproduction in any medium, provided the original author(s) and the source are credited.

\section{REFERENCES}

1. de Jong JC, Claas EC, Osterhaus AD, Webster RG, Lim WL. A pandemic warning? Nature. 1997;389(6651):554.

2. Neumann G, Noda T, Kawaoka Y. Emergence and pandemic potential of swine-origin H1N1 influenza virus. Nature. 2009;459(7249):931-9.

3. Gao R, Cao B, Hu Y, Feng Z, Wang D, Hu W, et al. Human infection with a novel avian-origin influenza A (H7N9) virus. N Engl J Med. 2013;368(20):1888-97.

4. Brown LE, Kelso A. Prospects for an influenza vaccine that induces cross-protective cytotoxic T lymphocytes. Immunol Cell Biol. 2009;87(4):300-8.

5. Sridhar S, Begom S, Bermingham A, Hoschler K, Adamson W, Carman W, et al. Cellular immune correlates of protection against symptomatic pandemic influenza. Nat Med. 2013;19(10):1305-12.

6. Stanekova Z, Vareckova E. Conserved epitopes of influenza A virus inducing protective immunity and their prospects for universal vaccine development. Virol J. 2010;7:351.

7. Assarsson E, Bui HH, Sidney J, Zhang Q Glenn J, Oseroff C, et al. Immunomic analysis of the repertoire of T-cell specificities for influenza A virus in humans. J Virol. 2008;82(24):12241-51.
8. Alexander J, Bilsel P, del Guercio MF, Marinkovic-Petrovic A, Southwood S, Stewart S, et al. Identification of broad binding class I HLA supertype epitopes to provide universal coverage of influenza A virus. Hum Immunol. 2010;71(5):468-74.

9. AmorijJP, Kersten GF, Saluja V, Tonnis WF, Hinrichs WL, Slutter $\mathrm{B}$, et al. Towards tailored vaccine delivery: needs, challenges and perspectives. J Control Release Off J Control Release Soc. 2012;161(2):363-76.

10. Arkema A, Huckriede A, Schoen P, Wilschut J, Daemen T. Induction of cytotoxic $\mathrm{T}$ lymphocyte activity by fusion-active peptide-containing virosomes. Vaccine. 2000;18(14):132733.

11. Amacker M, Engler O, Kammer AR, Vadrucci S, Oberholzer D, Gerny A, et al. Peptide-loaded chimeric influenza virosomes for efficient in vivo induction of cytotoxic $\mathrm{T}$ cells. Int Immunol. 2005;17(6):695-704.

12. Mallick AI, Parvizi P, Read LR, Nagy E, Behboudi S, Sharif S. Enhancement of immunogenicity of a virosome-based avian influenza vaccine in chickens by incorporating CpG-ODN. Vaccine. 201 1;29(8):1657-65.

13. de Jonge J, Amorij JP, Hinrichs WL, Wilschut J, Huckriede A, Frijlink HW. Inulin sugar glasses preserve the structural integrity and biological activity of influenza virosomes during freeze-drying and storage. Eur J Pharm Sci Off J Eur Fed Pharm Sci. 2007;32(1): $33-44$.

14. Stegmann T, Morselt HW, Booy FP, van Breemen JF, Scherphof G, Wilschut J. Functional reconstitution of influenza virus envelopes. EMBO J. 1987;6(9):2651-9.

15. Soema PG, Willems G-J, van Twillert K, van de Wijdeven G, Boog CJ, Kersten GFA, et al. Solid bioneedle-delivered influenza vaccines are highly thermostable and induce both humoral and cellular immune responses. PLoS One. 2014;9(3):e92806.

16. Wiesel M, Oxenius A. From crucial to negligible: functional CD8(+) T-cell responses and their dependence on CD4(+) T-cell help. Eur J Immunol. 2012;42(5):1080-8.

17. Li CK, Rappuoli R, Xu XN. Correlates of protection against influenza infection in humans-on the path to a universal vaccine? Curr Opin Immunol. 2013;25(4):470-6.

18. van de Sandt GE, Kreijtz JH, de Mutsert G, Geelhoed-Mieras MM, Hillaire ML, Vogelzang-van Trierum SE, et al. Human cytotoxic $\mathrm{T}$ lymphocytes directed to seasonal influenza a viruses cross-react with the newly emerging H7N9 virus. J Virol. 2014;88(3):1684-93.

19. Kammer AR, Amacker M, Rasi S, Westerfeld N, Gremion C, Neuhaus $\mathrm{D}$, et al. A new and versatile virosomal antigen delivery system to induce cellular and humoral immune responses. Vaccine. 2007;25(41):7065-74.

20. Poltl-Frank F, Zurbriggen R, Helg A, Stuart F, Robinson J, Gluck R, et al. Use of reconstituted influenza virus virosomes as an immunopotentiating delivery system for a peptide-based vaccine. Clin Exp Immunol. 1999;117(3):496-503.

21. Snapper CM, Paul WE. Interferon-gamma and B cell stimulatory factor-1 reciprocally regulate $\mathrm{Ig}$ isotype production. Science. 1987;236(4804):944-7.

22. Huber VC, McKeon RM, Brackin MN, Miller LA, Keating R, Brown SA, et al. Distinct contributions of vaccine-induced immunoglobulin G1 (IgG1) and IgG2a antibodies to protective immunity against influenza. Clin Vaccine Immunol CVI. 2006;13(9):98190.

23. Foged G, Hansen J, Agger EM. License to kill: Formulation requirements for optimal priming of CD8(+) CTL responses with particulate vaccine delivery systems. EurJ Pharm Sci OffJ Eur Fed Pharm Sci. 2012;45(4):482-91.

24. Budimir N, Meijerhof T, Wilschut J, Huckriede A, de Haan A. The role of membrane fusion activity of a whole inactivated influenza virus vaccine in (re)activation of 
influenza-specific cytotoxic $\mathrm{T}$ lymphocytes. Vaccine. 2010;28(52):8280-7.

25. Budimir N, Huckriede A, Meijerhof T, Boon L, Gostick E, Price DA, et al. Induction of heterosubtypic cross-protection against influenza by a whole inactivated virus vaccine: the role of viral membrane fusion activity. PLoS One. 2012;7(1):e30898.

26. Slutter B, Pewe LL, Kaech SM, Harty JT. Lung airway-surveilling CXCR3(hi) memory CD8(+) T cells are critical for protection against influenza A virus. Immunity. 2013;39(5):939-48.
27. Schotsaert M, Saelens X, Leroux-Roels G. Influenza vaccines: T-cell responses deserve more attention. Expert Rev Vaccines. 2012;11(8): 949-62.

28. Valkenburg SA, Li OT, Mak PW, Mok CK, Nicholls JM, Guan Y, et al. IL-15 adjuvanted multivalent vaccinia-based universal influenza vaccine requires CD4+ T cells for heterosubtypic protection. Proc Natl Acad Sci U S A. 2014;111(15):5676-81.

29. Pica N, Palese P. Toward a universal influenza virus vaccine: prospects and challenges. Annu Rev Med. 2013;64:189-202. 